\title{
Perceptions of Cannabis in sub-Saharan Africa: Findings from a general population survey in six African countries with different Cannabis policies.
}

Chenai Kitchen

Xi'an Jiaotong University

John Alimamy Kabba

Xi'an Jiaotong University

Tonny Ssekamatte

Makerere University

Ediomo-Ubong Nelson

University of Uyo

Samuel Adu-Gyamfi

Kwame Nkrumah University of Science and Technology

\section{Molewane Mametja}

University of the Witwatersrand

Caijun Yang

Xi'an Jiaotong University

Jie Chang

Xi'an Jiaotong University

Yu Fang ( $\nabla$ yufang@xjtu.edu.cn )

Xi'an Jiaotong University

Research Article

Keywords: Cannabis, sub-Saharan Africa, policy, perception

Posted Date: January 10th, 2022

DOI: https://doi.org/10.21203/rs.3.rs-1241985/v1

License: (c) (1) This work is licensed under a Creative Commons Attribution 4.0 International License. Read Full License 


\section{Abstract \\ Background}

Recently, legislative shifts in cannabis regulation away from exclusively prohibitionist controls have been seen in sub-Saharan African countries. However, the extent to which public perceptions are aligned with policy trends is unclear. Given that views concerning cannabis may be influenced by associated legislation we aimed to compare public opinion towards cannabis amongst countries with legislatively different cannabis policies.

\section{Methods}

We conducted an online survey of the general adult population in six purposively selected countries representative of maximum policy variation across subSaharan Africa i.e., Ghana, Nigeria, Sierra Leone, South Africa and Zimbabwe. Survey questions covered perceptions towards users, risks and benefits, regulations and public health impacts as well as sociodemographic characteristics. Chi square tests were used to analyse the association between categorical variables and explore country level differences.

\section{Results}

Totally 1216 responses were received. Large variations were noted in responses towards user stereotypes, risks and preferred legislation. In relation to users being honest $\left(X^{2}=43.316, P<0.0001\right)$, most in Sierra Leone(52.9\%), Nigeria (45.6\%) and Uganda(48.6\%) disagreed whereas in Ghana(39.4\%), South Africa(43.3\%) and Zimbabwe(40.1\%) most agreed. Differences in smoking cannabis and smoking tobacco products $\left(X^{2}=27.712, P=0.002\right)$ saw respondents from Ghana(51.4\%), Nigeria(58.8\%), Uganda(50.9\%) and Zimbabwe(46.8\%) agreeing that smoking cannabis is more harmful to health, whereas majorities in Sierra Leone(45.7\%) and South Africa(49.8\%) disagreed. Apart from South Africa were the greater proportion opted to allow cannabis for all purposes(28.8\%), majorities in other countries supported only medicinal legalisation $\left(X^{2}=96.631, P<0.0001\right)$.

\section{Conclusion}

Dependent upon the question of focus, at the liberal end of the policy spectrum are Ghana, South Africa and Zimbabwe whilst Nigeria, Sierra Leone and Uganda were more conservative. Responses tended to reflect the policy position of respective countries, however our findings suggest overall increasing medicinal cannabis support. Greater understanding of policy dynamics may help create frameworks for countries contemplating reforms.

\section{Introduction}

Cannabis, the most widely produced and consumed illicit drug, globally, is a generic term used to denote the several psychoactive preparations of the plant Cannabis sativa (Jain et al., 2018). The cultivation, trade and consumption of cannabis has been prohibited under international drug conventions for many years. Over the past decade, however, there have been calls to explore legal, decriminalised and commercial systems for production and use. The global drift towards more lenient approaches away from traditional prohibitionist controls are reflective of increased evidence supporting its efficacy, greater interest in cannabis-based therapeutics as well as, of its potentials for revenue generation and job creation. A number of countries and a growing cadre of US states have taken to the adoption of tailored approaches to cannabis regulation premised upon evidence-based and humane considerations(Manu et al., 2021).

Africa is a central hub for cannabis use with an estimated 13.2\% annual prevalence(Owusu et al., 2021). According to industry estimates, the continent annually produces a quarter of the global total (over 38,000tonnes) with countries such as Ghana, Nigeria and Eswatini being the most notable primary sources and trafficking points(Partners, 2019). Notwithstanding the increasing cannabis production and use in Sub-Saharan Africa, it has not been an exception to the wave of global reforms concerning policies on cannabis. Nations including, Lesotho, Malawi, Morocco, South Africa, Zambia, and Zimbabwe(Beadle, 2021) have taken to restructuring their policies by adopting legalization and commercialization approaches, with the expectations of reaping medical and economic benefits. Most recently, on June 28, 2021, Rwanda under Ministerial Order No 003/MoH/2021, legalized growing, processing, exporting and medical use of cannabis(Beadle, 2021). Even so, across Africa there is no harmonized drug law and given that nations largely possess autonomy over respective cannabis legislation, there is little consensus amongst countries on cannabis laws. Policies in respective countries have been an amalgamation of local mandates and stipulations of the International Drug Control Treaties of the 1961 Single Convention on Narcotics Drugs. Among other things, this treaty classifies cannabis as addictive, of little or no therapeutic value and illegal for all signatories states(Putri, 2020). Policies arising thereby have oscillated from liberal to punitive.

Given that, views concerning cannabis may be influenced by legislation in the respective country of residency(Mendiburo-Seguel et al., 2017; Steigerwald et al., 2020; Sznitman \& Bretteville-Jensen, 2015), we aimed to compare public opinion towards cannabis amongst African countries with legislatively different cannabis policies. Our objective was to assess how general adult populations in countries with different policies perceive cannabis in terms of users, risks and benefits, regulations and health impact. We hypothesize that cannabis-related stigma is greater in more stringent policy settings, in other words restrictive measures and negative stereotypes would be more common among less-liberal countries than among those with more liberal policies.

\section{Methods}

\section{Sampling Strategy}


The countries covered in this study, Ghana, Nigeria, Sierra Leone, South Africa and Zimbabwe, are signatories to all UN drug control treaties (Putri, 2020), and were purposively selected as representative of the maximum variation in cannabis policy across sub-Saharan Africa (Table 1). Adult residents (18years and older) of any of the selected countries, were eligible to participate. Participant sampling was stratified by respective country's cannabis policy alignment.

Table 1

Overview of cannabis policy in the six countries of study.

\begin{tabular}{|c|c|c|c|c|c|c|}
\hline & Nigeria & Ghana & South Africa & Sierra Leone & Uganda & Zimbabwe \\
\hline $\begin{array}{l}\text { Main } \\
\text { Recreational } \\
\text { Cannabis } \\
\text { Legislation }\end{array}$ & $\begin{array}{l}\text { The National Drug Law } \\
\text { Enforcement Degree, } 1990 \\
\text { (Now an Acts of } \\
\text { Parliament CAP N30 Laws } \\
\text { of the Federation of } \\
\text { Nigeria, 2004). }\end{array}$ & $\begin{array}{l}\text { Narcotics Control } \\
\text { Commission Act, } 2020 \text { (Act } \\
\text { 1019) (2020), }\end{array}$ & $\begin{array}{l}\text { Cannabis for } \\
\text { Private Purposes } \\
\text { Bill, } 2020\end{array}$ & $\begin{array}{l}\text { The National } \\
\text { Drugs Control } \\
\text { Act, } 2008\end{array}$ & $\begin{array}{l}\text { The Narcotic } \\
\text { Drugs and } \\
\text { Psychotropic } \\
\text { Substances } \\
\text { (Control) Act, } \\
2015\end{array}$ & $\begin{array}{l}\text { Chapter 15:02 } \\
\text { Dangerous Drugs } \\
\text { Act (as amended) }\end{array}$ \\
\hline $\begin{array}{l}\text { Main } \\
\text { Medicinal } \\
\text { Cannabis } \\
\text { Legislation }\end{array}$ & None & $\begin{array}{l}\text { Narcotics Control } \\
\text { Commission Act, } 2020 \text { (Act } \\
\text { 1019) (2020), }\end{array}$ & $\begin{array}{l}\text { Medicines and } \\
\text { Related } \\
\text { Substances Act } \\
\text { (Act } 101 \text { of } \\
\text { 1965) }\end{array}$ & $\begin{array}{l}\text { Guideline for } \\
\text { the } \\
\text { Cultivation } \\
\text { and } \\
\text { Processing of } \\
\text { Medical } \\
\text { Cannabis } \\
\text { (2019) }\end{array}$ & $\begin{array}{l}\text { The Narcotic } \\
\text { Drugs and } \\
\text { Psychotropic } \\
\text { Substances } \\
\text { (Control) Act, } \\
2015\end{array}$ & $\begin{array}{l}\text { Statutory } \\
\text { Instrument } 62 \text { of } \\
\text { 2018. [CAP. 15:02 } \\
\\
\text { Dangerous Drugs } \\
\text { (Production of } \\
\text { Cannabis for } \\
\text { Medicinal and } \\
\text { Scientific Use) } \\
\text { Regulations, } 2018\end{array}$ \\
\hline $\begin{array}{l}\text { Regulatory } \\
\text { body }\end{array}$ & $\begin{array}{l}\text { National Drug Law } \\
\text { Enforcement Agency } \\
\text { (NDLEA) }\end{array}$ & $\begin{array}{l}\text { Narcotics Control } \\
\text { Commission (NACOC) }\end{array}$ & $\begin{array}{l}\text { South African } \\
\text { Health Products } \\
\text { Authority } \\
\text { (SAHPRA) }\end{array}$ & $\begin{array}{l}\text { National Drug } \\
\text { Law } \\
\text { Enforcement } \\
\text { Agency } \\
\text { (NDLEA) }\end{array}$ & $\begin{array}{l}\text { National Drug } \\
\text { Authority } \\
\text { (NDA) }\end{array}$ & $\begin{array}{l}\text { Medicines Control } \\
\text { Authority of } \\
\text { Zimbabwe(MCAZ), } \\
\text { Ministry of Health } \\
\text { and Child Welfare }\end{array}$ \\
\hline $\begin{array}{l}\text { Recreational } \\
\text { Cannabis } \\
\text { Legal status }\end{array}$ & Illegal & Decriminalized & $\begin{array}{l}\text { Decriminalized } \\
\text { (Confined to } \\
\text { private settings } \\
\text { to which the } \\
\text { public does not } \\
\text { have access as } \\
\text { of right.) }\end{array}$ & Illegal & Illegal & Illegal \\
\hline $\begin{array}{l}\text { Medicinal } \\
\text { Cannabis } \\
\text { Legal status }\end{array}$ & Illegal & $\begin{array}{l}\text { Decriminalized (use and } \\
\text { cultivation) }\end{array}$ & $\begin{array}{l}\text { Legal for all } \\
\text { purposes }\end{array}$ & $\begin{array}{l}\text { Legal for } \\
\text { cultivation } \\
\text { purposes }\end{array}$ & Illegal & $\begin{array}{l}\text { Legal for cultivation } \\
\text { and research } \\
\text { purposes }\end{array}$ \\
\hline $\begin{array}{l}\text { Penalties } \\
\text { for } \\
\text { cannabis } \\
\text { possession } \\
\text { for personal } \\
\text { use }\end{array}$ & $\begin{array}{l}\text { Punishable by no less } \\
\text { than ten years } \\
\text { imprisonment }\end{array}$ & $\begin{array}{l}\text { Civil penalties i.e. fines of } \\
200-500 \text { penalty units (Ane, } \\
2020 \text { ) or treatment referrals. } \\
\text { Failure to pay fines, } \\
\text { translates into a } 15 \text { months } \\
\text { jail sentence. }\end{array}$ & $\begin{array}{l}\text { Public } \\
\text { consumption is } \\
\text { liable to a fine or } \\
\text { between two and } \\
\text { fifteen years of } \\
\text { imprisonment. }\end{array}$ & $\begin{array}{l}\text { Minimum of } \\
\text { five years in } \\
\text { imprisonment }\end{array}$ & $\begin{array}{l}\text { Liable to a } \\
\text { fine and or } \\
\text { one to five } \\
\text { years } \\
\text { imprisonment }\end{array}$ & $\begin{array}{l}\text { Possession or use } \\
\text { are subject to a fine } \\
\text { and or at least six } \\
\text { months } \\
\text { imprisonment or } \\
\text { court ordered } \\
\text { treatment. }\end{array}$ \\
\hline
\end{tabular}

On a spectrum from liberal to punitive, we placed the South Africa on the liberal side and Nigeria on the punitive side. According to South African legislation, cannabis was approved for medical purposes in 2017 and following a 2018 constitutional court ruling, which ruled cannabis criminalisation as breach to the right to privacy(Tharoor, 2018), private consumption and cultivation was decriminalised(AFRICA, 2020)(Veldman, 2020b). Ghana, passed the Narcotics Control Commission Act (Act 1019), 2020. This act essentially decriminalizes cannabis use for commercial and health purposes(Owusu et al., 2021), representing a country systematically aiming at liberalization and the next most liberal country in our study.

Zimbabwe and Sierra Leone take intermediate positions, as policies in both countries legalise cultivation for medicinal and research purposes but do not have provisions for legal recreational use or for patient access(Veldman, 2020a). However, in the former country there has been greater implementation as evidenced by government support and issuance of licences and ongoing medicinal cannabis activity(Partners, 2019; Sowoya et al., 2020), which for our purposes places it on the liberal half of the spectrum. Whereas for the latter, we labelled as more conversative owing to the fact that, plans to initiate cannabis related activities have been stagnated by multiple policy re-evaluations and widespread condemnation from religious, political and conservative groups(BENDU, 2017; Veldman, 2020a).

In Uganda, government institutions have taken a public prohibitionist position on drugs(Eligh, 2019) however the country can be classified as transitioning system, yet still closer to the punitive end. Recreational consumption and cultivation are stringently restricted but, the Narcotic Drugs and Psychotropic Substances Act, 2015, legalises production and exportation of medical cannabis, although guidelines for such activities have not been ratified(AFRICA, 2021; Partners, 2019). At the far end of spectrum, Nigeria which makes no distinction between recreational or medicinal cannabis with any associated activity regarded as illegal has the most punitive cannabis policy(Dirisu et al., 2018).

\section{Survey Instrument}

Survey questions were selected or adapted from previous public opinion and substance use research(Greenfield et al., 2004; Jain et al., 2018; Jasmine Turna, 2020; Lintzeris et al., 2020; Luty \& Grewal, 2002; Mikos \& Kam, 2019; Robertson et al., 2020; Turna et al., 2020; Williams et al., 2016), with input from relevant 
experts used to tailor questioning to country specific relevance. To further ensure validity and reliability a pilot study was conducted. Ten individuals from each country were invited to complete the survey and as a result, some questions were rephrased for clarity, grammatical errors corrected and additional options added. Data from the pilot study were excluded from the main study.

Our final questionnaire had six sections; 1)demographics: including age, sex, country, marital status, religion, educational level and employment status.; 2)cannabis use: considering ever cannabis use and awareness of a current user; 3 ) risks and benefits: including two multi-selection questions to pick from a list of potential risks and benefits and 5 point Likert statements comparing cannabis use against alcohol and tobacco; 4)cannabis laws: considering preferential choice of cannabis legal status and 5 point Likert scale statements on cannabis as a source of problems and as easily accessible; 5 )cannabis users: 5 point Likert scale statements used to consider user stereotypes of gender, morality /mental health status, work ethic and criminality and a single option question on how users should be addressed under the law and; 6 ) legalisation of cannabis: all 5 point Likert scale statements considering associated medical efficacy; impact on crime levels, employment opportunities and medical options; age restrictions; provision of information on safe use; and tolerance towards sale and public use.

\section{Data Collection}

The online cross-sectional survey was 'live' from October 22 to December 10, 2021. Data were collected via KoboToolbox, a secure web-based platform allowing respondents to directly enter responses online. The link was promoted using popular social media platforms i.e., Facebook and WhatsApp. Respondents read and agreed to an informed consent statement before filling in the survey. All of the data provided by respondents were anonymous and kept confidential.

\section{Statistical Analyses}

Analyses were conducted using Statistical Package for Social Sciences(SPSS) Statistics v.27. Likert-scale responses were aggregated into three categories i.e., agree, disagree and not sure. We compared countries using proportional analysis to summarize the demographic data. Cross tabulations and proportional analysis were used for multi-response questions. Chi square tests were used to analyse the association between categorical variables and explore the differences in perceptions towards cannabis use of among residents in countries with different policies. A $P$ value $<0.05$ was considered to be statistically significant.

\section{Ethical Considerations}

The study received ethics clearance from the Health Science Centre of Xi'an Jiaotong University. The survey tool is available as supplementary material (Supplementary File1).

\section{Results \\ Demographics}

Overall, 1216 responses were recorded with South Africa (18.6\%) registering the most responses, followed by Zimbabwe(18.3\%), Uganda(17.9\%), Ghana(17.1\%), Nigeria(16.8\%) and Sierra Leone(11.3\%). Most respondents were male, except in South Africa and Zimbabwe. The majority of respondents in all countries were aged between $18-30$ years(56.0\%) and Christians(65.5\%). In Nigeria most participants were married (49.0\%) whereas, in all other countries they were single, with Sierra Leone recording the highest number of single participants (76.8\%). Generally, most respondents were educated to the postgraduate level (50.7\%), with Uganda found to have the highest proportion of postgraduate degree holders(66.1\%), closely followed by Sierra Leone(63.8\%) and Nigeria(52.9\%). Students(26.5\%) made up the bulk of our sample group and were the largest represented group in Ghana(26.0\%), Sierra Leone(52.2\%) and Zimbabwe(25.7\%).In Nigeria and Uganda, respondents were mainly employed, (34.3\% and 38.1\% respectively), whereas in South Africa they were mostly unemployed(27.4\%).

An evident difference was shown in ever cannabis use $\left(X^{2}=16.148, P=0.006\right)$ and in knowing a current user $\left(X^{2}=51.402, P<0.0001\right)$. Nearly two-thirds $(62.7 \%)$ had never used cannabis however, a nearly similar proportion reported knowing a current user(63.1\%). On a country level, South Africa(48.2\%) had the highest proportion of people who have used cannabis and Sierra Leone had the lowest(29.7\%). Sierra Leone had the highest proportion of respondents aware of a current user(83.3\%), whilst over half of Zimbabweans(51.8\%) reported not knowing a current user. (Table 2) 
Table 2

Sociodemographic characteristics.

\begin{tabular}{|c|c|c|c|c|c|c|c|c|}
\hline COUNTRY n(\%) & $\begin{array}{l}\text { Total } \\
\mathrm{N}=1216\end{array}$ & $\begin{array}{l}\text { Ghana } \\
208(17.1)\end{array}$ & $\begin{array}{l}\text { Nigeria } \\
\text { 204(16.8) }\end{array}$ & $\begin{array}{l}\text { Sierra Leone } \\
\text { 138(11.3) }\end{array}$ & \multicolumn{2}{|c|}{$\begin{array}{l}\text { South Africa } \\
226(18.6)\end{array}$} & $\begin{array}{l}\text { Uganda } \\
218(17.9)\end{array}$ & $\begin{array}{l}\text { Zimbabwe } \\
\text { 222(18.3) }\end{array}$ \\
\hline \multicolumn{9}{|l|}{ GENDER } \\
\hline Male & $\begin{array}{l}627 \\
(51.6)\end{array}$ & $\begin{array}{l}104 \\
(50.0)\end{array}$ & $\begin{array}{l}108 \\
(52.9)\end{array}$ & $88(63.8)$ & $\begin{array}{l}111 \\
(49.1)\end{array}$ & $113(51.8)$ & & $103(46.4)$ \\
\hline Female & $\begin{array}{l}583 \\
(47.9)\end{array}$ & $\begin{array}{l}102 \\
(49.0)\end{array}$ & $96(47.1)$ & $48(34.8)$ & $\begin{array}{l}115 \\
(50.9)\end{array}$ & $103(47.2)$ & & $119(53.6)$ \\
\hline Prefer not to say & $6(0.5)$ & $2(1.0)$ & $0(0.0)$ & $2(1.4)$ & $0(0.0)$ & $2(0.9)$ & & $0(0.0)$ \\
\hline \multicolumn{9}{|l|}{ AGE, yrs } \\
\hline $18-30$ & $\begin{array}{l}681 \\
(56.0)\end{array}$ & $\begin{array}{l}103 \\
(49.5)\end{array}$ & $94(46.1)$ & $109(79.0)$ & $\begin{array}{l}122 \\
(54.0)\end{array}$ & $132(60.6)$ & & $121(54.5)$ \\
\hline $31-45$ & $\begin{array}{l}423 \\
(34.8)\end{array}$ & $78(37.5)$ & $86(42.2)$ & $25(18.1)$ & $82(36.3)$ & $74(33.9)$ & & $78(35.1)$ \\
\hline Above 45 & $112(9.2)$ & $27(13.0)$ & $24(11.8)$ & $4(2.9)$ & $22(9.7)$ & $12(5.5)$ & & $23(10.4)$ \\
\hline \multicolumn{9}{|l|}{ RELIGION } \\
\hline Christians & $\begin{array}{l}796 \\
(65.5)\end{array}$ & $\begin{array}{l}144 \\
(69.2)\end{array}$ & $\begin{array}{l}120 \\
(58.8)\end{array}$ & $75(54.3)$ & $\begin{array}{l}143 \\
(63.3)\end{array}$ & $160(73.4)$ & & $154(69.4)$ \\
\hline Muslims & $221(18.2)$ & $32(15.4)$ & 65 (31.9) & $62(44.9)$ & $15(6.6)$ & 38 (17.4) & & $9(4.1)$ \\
\hline Others & $\begin{array}{l}199 \\
(16.4)\end{array}$ & $32(15.4)$ & $19(9.3)$ & $1(0.7)$ & $68(30.1)$ & $20(9.2)$ & & $59(26.6)$ \\
\hline \multicolumn{9}{|l|}{ MARITAL STATUS } \\
\hline Single & $\begin{array}{l}641 \\
(52.7)\end{array}$ & $98(47.1)$ & $94(46.1)$ & $106(76.8)$ & $\begin{array}{l}115 \\
(50.9)\end{array}$ & $115(52.8)$ & & $113(50.9)$ \\
\hline $\begin{array}{l}\text { Married or domestic } \\
\text { partnership }\end{array}$ & $\begin{array}{l}481 \\
(39.6)\end{array}$ & $95(45.7)$ & $\begin{array}{l}100 \\
(49.0)\end{array}$ & $32(23.2)$ & $82(36.3)$ & $93(42.7)$ & & $79(35.6)$ \\
\hline Others & $94(7.7)$ & $15(7.2)$ & $10(4.9)$ & $0(0)$ & $29(12.8)$ & $10(4.6)$ & & $30(13.5)$ \\
\hline \multicolumn{9}{|l|}{ EDUCATIONAL LEVEL } \\
\hline Bachelor's degree and below & $\begin{array}{l}600 \\
(49.3)\end{array}$ & $\begin{array}{l}114 \\
(54.8)\end{array}$ & $96(47.1)$ & $50(36.2)$ & $\begin{array}{l}142 \\
(62.8)\end{array}$ & $74(33.9)$ & & $124(55.9)$ \\
\hline Postgraduate & $\begin{array}{l}616 \\
(50.7)\end{array}$ & $94(45.2)$ & $\begin{array}{l}108 \\
(52.9)\end{array}$ & $88(63.8)$ & $84(37.2)$ & $144(66.1)$ & & $98(44.1)$ \\
\hline \multicolumn{9}{|l|}{ EMPLOYMENT STATUS } \\
\hline Student & $\begin{array}{l}322 \\
(26.5)\end{array}$ & $54(26.0)$ & $42(20.6)$ & $72(52.2)$ & $48(21.2)$ & $49(22.5)$ & & $57(25.7)$ \\
\hline Unemployed & $\begin{array}{l}226 \\
(18.6)\end{array}$ & $32(15.4)$ & $29(14.2)$ & $18(13.0)$ & $62(27.4)$ & $33(15.1)$ & & $52(23.4)$ \\
\hline Self-employed & $\begin{array}{l}246 \\
(20.2)\end{array}$ & $52(25.0)$ & $41(20.1)$ & $10(7.2)$ & $44(19.5)$ & $45(20.6)$ & & $54(24.3)$ \\
\hline Employed & $\begin{array}{l}314 \\
(25.8)\end{array}$ & $47(22.6)$ & $70(34.3)$ & $36(26.1)$ & $48(21.2)$ & $83(38.1)$ & & $30(13.5)$ \\
\hline Others & $108(8.9)$ & $23(11.1)$ & $22(10.8)$ & $2(1.4)$ & $24(10.6)$ & $8(3.7)$ & & $29(13.1)$ \\
\hline \multicolumn{9}{|l|}{ Have you ever used cannabis? } \\
\hline No & $\begin{array}{l}762 \\
(62.7)\end{array}$ & $\begin{array}{l}131 \\
(63.0)\end{array}$ & $\begin{array}{l}133 \\
(65.2)\end{array}$ & $97(70.3)$ & $\begin{array}{l}117 \\
(51.8)\end{array}$ & $140(64.2)$ & & $144(64.9)$ \\
\hline Yes & $\begin{array}{l}454 \\
(37.3)\end{array}$ & $77(37.0)$ & $71(34.8)$ & $41(29.7)$ & $\begin{array}{l}109 \\
(48.2)\end{array}$ & $78(35.8)$ & & $78(35.1)$ \\
\hline \multicolumn{9}{|c|}{ Are you aware of a friend/family member or colleague who is a current cannabis user? } \\
\hline No & $\begin{array}{l}449 \\
(36.9)\end{array}$ & $67(32.2)$ & $88(43.1)$ & $23(16.7)$ & $80(35.4)$ & $76(34.9 \%)$ & & $115(51.8)$ \\
\hline Yes & $\begin{array}{l}767 \\
(63.1)\end{array}$ & $\begin{array}{l}141 \\
(67.8)\end{array}$ & $\begin{array}{l}116 \\
(56.9)\end{array}$ & $115(83.3)$ & $\begin{array}{l}146 \\
(64.6)\end{array}$ & $142(65.1)$ & & $107(48.2)$ \\
\hline
\end{tabular}


Respondents were questioned if users were mentally ill $\left(X^{2}=75.046, P<0.0001\right)$, to which, most disagreed with proportions ranging from nearly three quarters of Zimbabwean participants(72.1\%) to almost half in Sierra Leone(44.9\%). In relation to users being honest $\left(X^{2}=43.316, P<0.0001\right)$, most in Sierra Leone(52.9\%), Nigeria (45.6\%) and Uganda(48.6\%) disagreed, and larger proportions in Ghana(39.4\%), South Africa(43.3\%) and Zimbabwe(40.1\%) agreed. Around half of participants in Ghana and South Africa and Zimbabwe agreed to users being hardworking whereas majorities in Uganda, Sierra Leone and Nigeria expressed disagreement $\left(X^{2}=48.567, P<0.0001\right)$. In terms of gender (i.e., users being mostly male), although statistically significant $\left(X^{2}=63.494\right.$, $P<0.0001)$, there was no distinction amongst countries with over sixty percent agreement. In all cases large proportions disagreed to the idea that users are criminals, with South Africa having the largest proportion (75.7\%) and Sierra Leone the lowest $(50.0 \%),\left(X^{2}=74.024, P<0.0001\right)$. (Table 3)

Table 3

Perceptions of Cannabis users.

\begin{tabular}{|c|c|c|c|c|c|c|c|c|c|}
\hline STATEMENT & TOTAL & $\begin{array}{l}\text { Ghana } \\
(208)\end{array}$ & $\begin{array}{l}\text { Nigeria } \\
(204)\end{array}$ & $\begin{array}{l}\text { Sierra Leone } \\
(138)\end{array}$ & $\begin{array}{l}\text { South Africa } \\
\text { (226) }\end{array}$ & $\begin{array}{l}\text { Uganda } \\
(218)\end{array}$ & $\begin{array}{l}\text { Zimbabwe } \\
\text { (222) }\end{array}$ & $\mathrm{Chi}^{2} / \mathrm{F}(\mathrm{df})$ & $\mathbf{P}$ \\
\hline \multicolumn{6}{|c|}{ I think most users are male } & & & $\begin{array}{l}63.494 \\
(10)\end{array}$ & $<.0001$ \\
\hline AGREE & $810(66.6)$ & $137(65.9)$ & $129(63.2)$ & $103(74.6)$ & $145(64.2)$ & $140(64.2)$ & $156(70.3)$ & & \\
\hline DISAGREE & $333(27.4)$ & $61(29.3)$ & $\begin{array}{l}6732.8 \\
(32.8)\end{array}$ & $17(12.3)$ & $75(33.2)$ & $50(22.9)$ & $63(28.4)$ & & \\
\hline NOT SURE & $73(6)$ & $10(4.8)$ & $8(3.9)$ & 18(13.0) & $6(2.7)$ & $28(12.8)$ & $3(1.4)$ & & \\
\hline \multicolumn{8}{|c|}{ I think most users are hardworking and focused } & $48.576(10)$ & $<0.0001$ \\
\hline AGREE & $490(40.3)$ & $105(50.5)$ & $78(38.2)$ & $34(24.6)$ & $116(51.3)$ & $66(30.3)$ & $91(41.0)$ & & \\
\hline DISAGREE & $478(39.3)$ & $65(31.3)$ & $90(44.1)$ & $64(46.4)$ & 75(33.2) & 103(47.2) & $81(36.5)$ & & \\
\hline NOT SURE & $248(20.4)$ & $38(18.3)$ & $36(17.6)$ & $40(29.0)$ & $35(15.5)$ & $49(22.5)$ & $50(22.5)$ & & \\
\hline \multicolumn{8}{|c|}{ I think most users are mentally ||| or immoral (sinners) } & $75.046(10)$ & $<0.0001$ \\
\hline AGREE & $321(26.4)$ & $46(22.1)$ & $59(28.9)$ & $49(35.5)$ & $58(25.7)$ & $56(25.7)$ & $53(23.9)$ & & \\
\hline DISAGREE & $758(62.3)$ & $143(68.8)$ & $124(60.8)$ & $62(44.9)$ & $156(69)$ & $113(51.8)$ & $160(72.1)$ & & \\
\hline NOT SURE & 137(11.3) & 19(9.1) & $21(10.3)$ & $27(19.6)$ & $12(5.3)$ & $49(22.5)$ & $9(4.1)$ & & \\
\hline \multicolumn{8}{|c|}{ I think most users are trustworthy and honest } & $43.316(10)$ & $<.0001$ \\
\hline AGREE & $418(34.4)$ & $82(39.4)$ & $61(29.9)$ & $26(18.8)$ & $98(43.4)$ & $62(28.4)$ & $89(40.1)$ & & \\
\hline DISAGREE & $495(40.7)$ & $65(31.3)$ & $93(45.6)$ & $73(52.9)$ & $80(35.4)$ & $106(48.6)$ & $78(35.1)$ & & \\
\hline NOT SURE & $303(24.9)$ & $61(29.3)$ & $50(24.5)$ & $39(28.3)$ & $48(21.2)$ & $50(22.9)$ & $55(24.8)$ & & \\
\hline \multicolumn{8}{|c|}{ I think most users are criminals } & $74.024(10)$ & $<.0001$ \\
\hline AGREE & 291(23.9) & $44(21.2)$ & $42(20.6)$ & $43(31.2)$ & $48(21.2)$ & $62(28.4)$ & $52(23.4)$ & & \\
\hline DISAGREE & $789(64.9)$ & $151(72.6)$ & $134(65.7)$ & $69(50.0)$ & $171(75.7)$ & $110(50.5)$ & $154(69.4)$ & & \\
\hline NOT SURE & $136(11.2)$ & $13(6.3)$ & 28(13.7) & $26(18.8)$ & $7(3.10$ & $46(21.1)$ & $16(7.2)$ & & \\
\hline
\end{tabular}

\section{Risks and Benefits}

Proportional analyses showed that perceptions of associated health, social and substance abuse risks were similar across countries (Figure 1 (a)). The belief that use leads to substance abuse, addiction and polysubstance use was recognised by nearly a quarter of all respective country participants, with Uganda(31.1\%) and Nigeria(31.0\%) having the highest proportions. In terms of perceived benefits of use, Figure 1(b) shows that Ghana(55.4\%) and South Africa(55.4\%) had larger proportions of participants who endorsed the belief that cannabis use has health benefits. Cross tabulation also revealed that, compared to other nationalities, South Africans(35\%) more greatly considered cannabis to have social benefits. The belief that cannabis use is non-beneficial was most supported by Sierra Leoneans(25.9\%) and least supported by South Africans(8.4\%).(Figure 1)

\section{Public health domains}

There were statistically significant differences in comparisons between smoking cannabis and smoking tobacco products $\left(X^{2}=27.712, P=0.002\right)$. Respondents from Ghana, Nigeria, Uganda and Zimbabwe agreed that smoking cannabis is more harmful to health than smoking tobacco products, whilst nearly half of Sierra Leoneans(45.7\%) and South Africans(49.8\%) disagreed. The greater part of participants in all countries disagreed with public use. Overwhelming majorities in all countries indicated being comfortable with medical cannabis sales at health facilities $\left(X^{2}=27.572, P=0.002\right)$. Responses regarding the comparison of driving under the influence of cannabis as safer than driving under the influence of alcohol showed statistically significant variation $\left(X^{2}=38.597, P<0.0001\right)$. Most participants in Ghana, Nigeria, Uganda and Zimbabwe disagreed, conversely, most from Sierra Leone and South Africa(42.0\% and $51.8 \%$ ) agreed. The use of cannabis as a safe and efficacious medicine was largely agreed upon by most in all countries, highest being in Uganda(60.1\%) 
and least by Ghana $(47.1 \%)\left(X^{2}=33.978, P<0.0001\right)$. Respondents did support the idea that medical use should only be permissible for specific problems by a medical professional $\left(X^{2}=27.712, P=0.02\right)$. (Table 4)

Table 4

Opinions regarding public health domains.

\begin{tabular}{|c|c|c|c|c|c|c|c|c|c|}
\hline STATEMENT & TOTAL & $\begin{array}{l}\text { Ghana } \\
\text { (208) }\end{array}$ & $\begin{array}{l}\text { Nigeria } \\
(204)\end{array}$ & Sierra Leone (138) & South Africa (226) & $\begin{array}{l}\text { Uganda } \\
\text { (218) }\end{array}$ & Zimbabwe (222) & $\mathrm{Chi}^{2} / \mathrm{F}(\mathrm{df})$ & $\mathbf{P}$ \\
\hline \multicolumn{8}{|c|}{ Smoking cannabis is more harmful to health than smoking cigarettes/ tobacco. } & $46.504(10)$ & $<.0001$ \\
\hline AGREE & $597(49.1)$ & $107(51.4)$ & $120(58.8)$ & $57(41.3)$ & $98(43.4)$ & $111(50.9)$ & 104(46.8) & & \\
\hline DISAGREE & $477(39.2)$ & $79(38.0)$ & $69(33.8)$ & $63(45.7)$ & $112(49.6)$ & $62(28.4)$ & $92(41.4)$ & & \\
\hline NOT SURE & $142(11.7)$ & $22(10.6)$ & $15(7.4)$ & $18(13.0)$ & $16(7.1)$ & $45(20.6)$ & 26(11.7) & & \\
\hline \multicolumn{8}{|c|}{ Driving under the influence of cannabis is safer than driving under the influence of alcohol. } & $38.597(10)$ & $<.0001$ \\
\hline AGREE & $492(40.5)$ & $89(42.8)$ & 69(33.8) & $58(42.0)$ & $117(51.8)$ & $65(29.8)$ & $94(42.3)$ & & \\
\hline DISAGREE & $560(46.1)$ & $94(45.2)$ & $112(54.9)$ & $53(38.4)$ & $91(40.3)$ & $113(51.8)$ & $97(43.7)$ & & \\
\hline NOT SURE & 164(13.5) & $25(12.0)$ & $23(11.3)$ & $27(19.6)$ & $18(8.0)$ & $40(18.3)$ & $31(14.0)$ & & \\
\hline \multicolumn{8}{|c|}{ Using cannabis as medicine is safe and effective. } & $33.978(10)$ & $<.0001$ \\
\hline AGREE & $655(53.9)$ & $98(47.1)$ & $107(52.5)$ & $79(57.2)$ & $128(56.6)$ & $131(60.1)$ & $112(50.5)$ & & \\
\hline DISAGREE & $321(26.4)$ & $57(27.4)$ & $72(35.3)$ & $27(19.6)$ & $66(29.2)$ & $45(20.6)$ & $54(24.3)$ & & \\
\hline NOT SURE & $240(19.7)$ & $53(25.5)$ & $25(12.3)$ & $32(23.2)$ & $32(14.2)$ & 42(19.3) & $56(25.2)$ & & \\
\hline \multicolumn{8}{|c|}{ Using cannabis as medicine should only be allowed by a doctor for certain problems. } & $27.717(10)$ & .002 \\
\hline AGREE & $814(66.9)$ & $145(69.7)$ & $126(61.8)$ & 107(77.5) & $137(60.6)$ & $152(69.7)$ & $147(66.2)$ & & \\
\hline DISAGREE & $308(25.3)$ & $49(23.6)$ & $66(32.4)$ & 19(13.8) & $75(33.2)$ & $46(21.1)$ & $53(23.9)$ & & \\
\hline NOT SURE & $94(7.7)$ & $14(6.7)$ & $12(5.9)$ & $12(8.7)$ & $14(6.2)$ & $20(9.2)$ & $22(9.9)$ & & \\
\hline \multicolumn{8}{|c|}{ I would be comfortable with hospitals and pharmacies selling cannabis products (capsules, oils) to patients? } & $27.572(10)$ & .002 \\
\hline AGREE & $780(64.1)$ & $151(72.6)$ & $109(53.4)$ & $85(61.6)$ & $159(70.4)$ & $137(62.8)$ & $139(62.6)$ & & \\
\hline DISAGREE & $309(25.4)$ & $47(22.6)$ & $67(32.8)$ & $32(23.2)$ & $49(21.7)$ & $55(25.2)$ & $59(26.6)$ & & \\
\hline NOT SURE & $127(10.4)$ & $10(4.8)$ & $28(13.7)$ & 21(15.2) & 18(8.0) & 26(11.9) & $24(10.8)$ & & \\
\hline \multicolumn{8}{|c|}{ I would be comfortable with people using cannabis in public (parks, restaurants)? } & $58.318(10)$ & $<.0001$ \\
\hline AGREE & $274(22.5)$ & $48(23.1)$ & $34(16.7)$ & $21(15.2)$ & $80(35.4)$ & $37(17.0)$ & $54(24.3)$ & & \\
\hline DISAGREE & $740(60.9)$ & $123(59.1)$ & $139(68.1)$ & $98(71.0)$ & 110(48.7( & $157(72.0)$ & $113(50.9)$ & & \\
\hline NOT SURE & $202(16.6)$ & $37(17.8)$ & $31(15.2)$ & 19(13.8) & $36(15.9)$ & $24(11.0)$ & $55(24.8)$ & & \\
\hline
\end{tabular}

\section{Regulation}

Figure 2a shows overall, there was more support for legalising medicinal use than for complete legalisation. Differences between countries are statistically significant $\left(X^{2}=96.631, P<0.0001\right)$. Apart from South Africa, were greater proportions $(28.8 \%)$ opted to have cannabis allowed for all purposes, in all other countries majorities selected the option to allow for medical purposes only. Responses regarding the form of treatment towards users varied by country $\left(X^{2}=\right.$ $97.375, P<0.0001)$, with the two most popular being sending users to treatment and leaving users alone. Participants from Ghana, South Africa and Zimbabwe were more likely to endorse that users should be left alone whilst in Nigeria, Sierra Leone and Uganda the most felt they should be sent to treatment (Figure 2b).(Figure 2)

Participants were in agreement with cannabis being easily accessible with South Africa recording the highest proportion (80.1\%). A relationship exists between respondent's country and holding the view that cannabis use is a source of problems $\left(X^{2}=97.654, P<0.0001\right)$, as evidenced by large variations across country responses. Nigerian, Sierra Leonean and Uganda respondents considered the cannabis crop as problematic whereas most participants in Ghana, South Africa and Zimbabwe considered cannabis not a problem. Table 5 illustrates that in all countries most agreed that cannabis should only be sold to adults $\left(X^{2}=22.723, P=0.12\right)$. In all regions, majorities saw a direct connection between legalisation and increased crime $\left(X^{2}=22.2, P=0.01\right)$. Most participants in each country believed that cannabis legalisation can lead to the creation of business and employment activities, most notably in Ghana(78.8\%) and South Africa(75.2\%) $\left(X^{2}=58.105, P<0.0001\right)$. Regarding other relevant regulatory concerns, although not statistically significant, most agreed that: legalisation will give patients more medical options and countries that allow cannabis use should give information on laws and safe use.(Table 5) 
Table 5

Attitudes towards regulation of Cannabis.

\begin{tabular}{|c|c|c|c|c|c|c|c|c|c|}
\hline STATEMENT & TOTAL & $\begin{array}{l}\text { Ghana } \\
(208)\end{array}$ & $\begin{array}{l}\text { Nigeria } \\
(204)\end{array}$ & $\begin{array}{l}\text { Sierra Leone } \\
\text { (138) }\end{array}$ & $\begin{array}{l}\text { South Africa } \\
(226)\end{array}$ & $\begin{array}{l}\text { Uganda } \\
(218)\end{array}$ & $\begin{array}{l}\text { Zimbabwe } \\
\text { (222) }\end{array}$ & $\mathrm{Chi}^{2} / \mathrm{F}(\mathrm{df})$ & $\mathbf{P}$ \\
\hline \multicolumn{8}{|c|}{ I think cannabis (use, trade or cultivation) is a problem in my country. } & $97.654(10)$ & $<.0001$ \\
\hline AGREE & $562(46.2)$ & $80(38.5)$ & $110(53.9)$ & $101(73.2)$ & $91(40.3)$ & $97(44.5)$ & $83(37.4)$ & & \\
\hline DISAGREE & $530(43.6)$ & $114(54.8)$ & 77(37.7) & 21(15.2) & $125(55.3)$ & $85(39.0)$ & $108(48.6)$ & & \\
\hline NOT SURE & $124(10.2)$ & $14(6.7)$ & $17(8.3)$ & $16(11.6)$ & $10(4.4)$ & $36(16.5)$ & $31(14.0)$ & & \\
\hline \multicolumn{8}{|c|}{ Cannabis is easy to get in my country. } & $57.13(10)$ & $<.0001$ \\
\hline AGREE & $847(69.7)$ & $154(74.0)$ & $152(74.5)$ & $96(69.6)$ & $181(80.1)$ & $114(52.3)$ & $150(67.6)$ & & \\
\hline DISAGREE & 221(18.2) & 33(15.9) & $34(16.7)$ & $29(21.0)$ & 31(13.7) & $52(23.9)$ & $42(18.9)$ & & \\
\hline NOT SURE & $148(12.2)$ & 21(10.1) & $18(8.8)$ & $13(9.4)$ & $14(6.2)$ & $52(23.9)$ & $30(13.5)$ & & \\
\hline \multicolumn{8}{|c|}{ Legalising cannabis leads to more crime and use. } & $22.2(10)$ & .014 \\
\hline AGREE & $595(48.9)$ & $96(46.2)$ & $107(52.5)$ & $81(58.7)$ & $103(45.6)$ & $111(50.9)$ & $97(43.7)$ & & \\
\hline DISAGREE & $443(36.4)$ & $84(40.4)$ & 79(38.7) & $32(23.2)$ & $89(39.4)$ & 73(33.5) & $86(38.7)$ & & \\
\hline NOT SURE & $178(14.6)$ & $28(13.5)$ & $18(8.8)$ & $25(18.1)$ & $34(15.0)$ & $34(15.6)$ & $39(17.6)$ & & \\
\hline \multicolumn{8}{|c|}{ Legalising cannabis creates business and employment opportunities. } & $58.105(10)$ & $<.0001$ \\
\hline AGREE & $830(68.3)$ & 164(78.8) & $137(67.2)$ & $64(46.4)$ & $170(75.2)$ & $143(65.6)$ & $152(68.5)$ & & \\
\hline DISAGREE & $270(22.2)$ & $36(17.3)$ & $46(22.5)$ & $52(37.7)$ & $46(20.4)$ & $50(22.9)$ & $40(18.0)$ & & \\
\hline NOT SURE & $116(9.5)$ & $8(3.8)$ & 21(10.3) & $22(15.9)$ & $10(4.4)$ & $25(11.5)$ & $30(13.5)$ & & \\
\hline \multicolumn{8}{|c|}{ Legalising cannabis will give patients more medical options. } & $17.123(10)$ & .072 \\
\hline AGREE & $714(58.7)$ & $129(62.0)$ & $113(55.4)$ & $65(47.1)$ & $139(61.5)$ & $129(59.2)$ & $139(62.6)$ & & \\
\hline DISAGREE & $314(25.8)$ & $50(24.0)$ & $64(31.4)$ & $45(32.6)$ & $54(23.9)$ & $48(22.0)$ & $53(23.9)$ & & \\
\hline NOT SURE & $188(15.5)$ & 29(13.9) & $27(13.2)$ & $28(20.3)$ & $33(14.6)$ & $41(18.8)$ & $30(13.5)$ & & \\
\hline \multicolumn{8}{|c|}{ Countries that allow cannabis use should only sell to adults ( 18 years + ) } & $22.723(10)$ & .012 \\
\hline AGREE & 1048(86.2) & 188(90.4) & $173(84.8)$ & 114(82.6) & 200(88.5) & $171(78.4)$ & 202(91.0) & & \\
\hline DISAGREE & 112(9.2) & $14(6.7)$ & $22(10.8)$ & 15(10.9) & 18(8.0) & 29(13.3) & $14(6.3)$ & & \\
\hline NOT SURE & $56(4.6)$ & $6(2.9)$ & $9(4.4)$ & $9(6.5)$ & $8(3.5)$ & 18(8.3) & $6(2.7)$ & & \\
\hline \multicolumn{8}{|c|}{ Countries that allow cannabis use should give information on laws and safe use. } & $12.496(10)$ & .253 \\
\hline AGREE & 1065(87.6) & $178(85.6)$ & $174(85.3)$ & $121(87.7)$ & $204(90.3)$ & 192(88.1) & 196(88.3) & & \\
\hline DISAGREE & $102(8.4)$ & $23(11.1)$ & $22(10.8)$ & $7(5.1)$ & $17(7.5)$ & $15(6.9)$ & 18(8.1) & & \\
\hline NOT SURE & $49(4.0)$ & $7(3.4)$ & $8(3.9)$ & $10(7.2)$ & $5(2.2)$ & $11(5.0)$ & $8(3.6)$ & & \\
\hline
\end{tabular}

\section{Discussion}

Across the included countries, legislative trends are not uniformly reflected in public attitudes towards cannabis. Variations were noted in responses towards user stereotypes, cannabis being a problem, comparisons with tobacco and alcohol use, preferred legislation, and strategies towards addressing users. Consistent with our hypothesis, countries with more liberal policies were associated with more positive sentiments. Dependent upon the question of focus, at the liberal end of the spectrum are Ghana, South Africa and Zimbabwe whilst Nigeria, Sierra Leone and Uganda were more conservative. Questions were marked consistency across countries were identified related to the accessibility, age limits for sales, comfortability with regulated medical use and sale, public use and associated risks and benefits. Participants were asked if they had ever used cannabis and if they were aware of a current user, to better contextualise interpretation of attitudinal differences between countries. There is a relationship between toleration of cannabis and its consumption and awareness of current users: countries that are more liberal tended to have higher rates of ever cannabis use (above 35\%) whilst less liberal countries had higher rates of knowing a current user (above 56\%). These results indicate that country differences in cannabis use experience and encounters with users can be reflective in collective attitudes.

Overall, participants from more conservative countries more greatly endorsed common gender (male) and negative typecasts of users (criminals, mentally ill) and disagreed with positive personality attributes (hardworking and trustworthy). Studies have shown that African cultures and religions tend to consider cannabis consumption as taboo (Carrier \& Klantschnig, 2017; Mametja \& Ross, 2020) and our study found higher levels of religiosity amongst countries considered more conservative- particularly Sierra Leone. Cannabis use is a stigmatized behaviour associated with devaluation of users(Skliamis et al., 2020). 
Amongst users this could be linked to the need of concealment to avoid prejudice and punishment, particularly in countries such as Nigeria with harsh penalties for users(Nelson, 2018) and Uganda where harsher repealed law provisions are used to charge and prosecute users((HRAPF), 2015).Moreover, such divisions of stereotypes can provide an explanatory basis for the fact that participants in more liberal countries mainly considered that users should be left alone whereas sending them to treatment (medical or religious) was more greatly associated with less liberal countries.

Risk (social, health and substance abuse) perceptions were similar across countries. However, participants from more liberal countries had an overall more favourable view towards social and health benefits of cannabis use. Cannabis is typically introduced to adolescents in social settings such as parties (Mehanović et al., 2020) and moderate use is linked to positive social attributes whereas abstinence is viewed negatively(Robertson et al., 2020). As most survey participants are considered young this may explain the endorsement of social benefits. However, pressure to conform to socializing norms and engage in risky behaviours(Mametja \& Ross, 2020) equally explains why nearly similar proportions also believed cannabis to pose a social risk. The support of cannabis health benefits being greater than risks is consistent with previous research indicating a minimization in associated risk perception in European and American settings(Piontek et al., 2013; Steigerwald et al., 2020; Tamson et al., 2021). A possible explanation for perceived health benefits has been defined as "legitimacy-conferring process" whereby regardless of personal beliefs, medicinal cannabis is legitimized through expert backing from established health institutions(Sznitman \& Bretteville-Jensen, 2015), as is the case with the Medicines and Control Authority of Zimbabwe(MCAZ) or the South African Health Products Regulatory Authority(SAPHRA).

Regarding smoking cannabis as more harmful to health than tobacco products and driving under the influence of cannabis as safer than under the influence of alcohol, a divide was seen in responses with Sierra Leonean and South African respondents being more likely to believe cannabis use as safer in both instances. Although considered to be on different sides of the policy spectrum, these responses may be connected to experiential encounters with cannabis and users, given the fact that South Africa reported the highest proportion of ever cannabis use and Sierra Leone had the large percentage of participants aware of current users. Similar to previous studies that found enhanced tolerance for cannabis when purposed for medical reasons(Mikos \& Kam, 2019), majorities in all countries considered the sale and use of medicinal cannabis under doctor authorisation acceptable, safe and effective. This somewhat supports the assumption that public opinion toward cannabis is driven by deliberations related to medical effects, as opposed to current polices and concerns for detrimental public health effects(Sznitman \& Bretteville-Jensen, 2015). Evidence suggests that exclusive cannabis smokers, while, exposed to lower levels of harmful constituents than exclusive tobacco smokers and co-users, are still exposed to higher toxicant levels than non-smokers(Meier et al., 2021). Associations have been found between blood tetrahydrocannabinol(THC) levels and impaired performance with cannabis intoxication related to a statistically significant increased risk of vehicular accidents(Jasmine Turna, 2020). As policies evolve, the perspective that cannabis use is safe and of low risk may become a more salient public health discussion requiring targeted campaigns against misconceptions.

Support for legalization of cannabis was mainly associated with medical use. Total legalisation was less supported which may be linked to the idea that full legalisation would increase crime and illicit use as majorly agreed upon in all countries. While the consequences of liberalised cannabis systems on crime trends are not fully understood, there have been reports of increased illicit use and poisoning in jurisdictions with commercialized medical cannabis(Sznitman \& Bretteville-Jensen, 2015). This may explain why our participants, regardless of nationality, expressed the need for age restrictions and providing information on laws and safe use. Although contrasting to results from a similar Latin American study where support was largely associated with legalization of recreational use, our findings showcase the effect of territorial differences on public opinions and the importance of accurate assessments of current situations in respective countries.

Support for medical cannabis was further shown by most participants in agreement with legalization leading to greater medical options and employment opportunities. Countries are eyeing the socioeconomic prospect of legally regulating cannabis for medicinal, industrial and scientific purposes(Bizimungu, 2020; Bloomer, 2019; Partners, 2019). By 2023, a fully legal and regulated African cannabis industry, valued at over $\$$ US7.1b(Partners, 2019), is anticipated to boost regional employment particularly to the benefit of South Africa and Nigeria with the world's highest and third highest unemployment rate(Naidoo, 2021). Endorsements for medical applicability may also stem from reported successes of locally sourced plant-based medicines such as African Potato (Hypoxis spp) and Moringa for HIV positive patients, for which reputation alone was sufficient to establish a significant industry in Zimbabwe(Chisaka, 2019; Onifade \& Sciences, 2020; Salehi et al., 2018). Also, with imports comprising upwards of 70\% of drugs consumed in most sub-Saharan Africa countries(Conway et al., 2020), patients typically lack access to locally manufactured and affordable drugs. Increased pharmaceutical activity can improve medicines availability resulting to better health(Abbott et al., 2021).

We examined perceptions of formal controls by investigating cannabis as a source of problems and its ease of accessibility. The reported apparent ease in more conservative countries affirms their problem belief statement. Interestingly, liberal countries expressed that although easy to access, the trade does not stand as a problem. Cannabis ease of acquisition and availability is not surprising since it is the most used psychoactive drugs(Chan et al., 2018; Gebremariam et al., 2018; Peltzer \& Pengpid, 2018) and a United Nations report of ten surveyed African countries, highlighted its accessibility through findings of cannabis being considerably cheaper than bottled beer(Carrier \& Klantschnig, 2017). Disparities in the problematic perception of cannabis align with our theory and may speak to cannabis's ambiguity as a hard or soft drug, referred to as "quasilegality", which allows laws to be leniently or strictly enforced(Carrier \& Klantschnig, 2017). For instance, seizures and arrests are likely a means of extracting bribes, or increasing arrest quotas, than clear evidence of policy aligned prohibition(Eligh, 2019). Associations with narcotics may permeate into the cannabis trade. Data indicate growing heroin and cocaine trafficking networks linking South America to European markets via West Africa(United Nations Office on Drugs and Crime, 2019). Increase in trade activities and related arrests can feed into the perception of illegality and problematic among law enforcement as well as the general population.(MendiburoSeguel et al., 2017).

Policies have been concluded as crucial in the construction of cannabis perceptions and experiences (Skliamis et al., 2020). Furthermore, as public backing can impact and simultaneously be impacted by policies, it was imperative to conduct this study across different policy settings, to investigate whether public opinion differences were reflective of policy variations. Unlike other countries(Aliekperova et al., 2020; Resko et al., 2019), individuals in many African countries

Page $9 / 14$ 
are unable to vote for their favoured policy and changes are made largely without public consideration. The significances of policy implementation without formulation of public consensus have not been explored and should stand as a key area for countries considering similar actions.

\section{Limitations}

Owing to particular limitations, findings must be interpreted with caution. Firstly, the African population is over a billion and we included only six out of a total of 54 African countries therefore results cannot be generalized. The national representativeness of our survey also comes into question as we did not consider specific regional variations and settings which may have affected perceptions. The use of an internet survey may have limited responses to those with internet access and the population who chose to participate may differ from individuals who chose not to or were unable to. In our case most of our respondents were young and well-educated and therefore may have biased our findings to the opinions of this particular subgroup. We did not consider country level factors such as crime and violence indices associated with cannabis which may be pertinent. Data collection reliant upon self- reporting is subject to social desirability bias which may have made individuals less likely to report substance use behaviors; however, use of an anonymous survey should have mitigated this effect. Lastly, the study was cross-sectional therefore, it is beyond the ambit of this paper to assess if legislative reform drives public opinion, or vice versa. Future studies should consider examining existing perceptions of cannabis using greater experimental variation of question format, as well as incorporating qualitative components.

\section{Conclusion}

Amongst sub-Saharan African countries there exist significant differences in cannabis use and associated policy attitudes, suggesting that people are questioning the status quo. Respective country's policy and associated stringency appeared to be reflected on participants' responses. Our findings imply increasing public support for medical cannabis whilst recreational use remains a more contentious issue. This is based on the observation that there is a very clear direction of travel amongst countries towards forms of cannabis liberalisation with the main underpinning argument for reform in both conservative and liberal countries being the potential medicinal and economic benefits afforded by less stringent regulations. It is essential to gain a more nuanced understanding of policy dynamics as well as the related degree and rationale for public opinion as even conservative countries appeared on certain topics to be divided. This may benefit countries contemplating reformation.

\section{Declarations}

\section{Author Contributions}

CK: Conceptualization, Methodology, Formal analysis, Writing- Original draft preparation. JAK: Conceptualization, Writing- Reviewing and Editing. TS, EN, SA, MM: Conceptualization, Data Collection, Reviewing. YF, JC, CY: Conceptualization, Supervision.

\section{Declarations of interest: None}

\section{Acknowledgements}

None

Funding

This research was supported by the "Young Talent Support Plan, "High Achiever Plan" of Health Science Centre, Xi'an Jiaotong University, and the Central University Basic Research Fund (2015qngz05).

\section{Ethical Considerations}

The study received ethics clearance from the Health Science Centre of Xi'an Jiaotong University. Consent was obtained from every participant before taking part in the survey and ensure confidentiality, no information related to respondent identity was collected.

\section{Availability of data}

Datasets generated during this current study are available from the corresponding author on reasonable request.

\section{References}

1. (HRAPF), H. R. A. a. P. F. (2015). THE NARCOTIC DRUGS AND PSYCHOTROPIC SUBSTANCES (CONTROL) ACT, 2015 AND THE LEGAL REGULATION OF DRUG USE IN UGANDA. https://hrapf.org/index.php/resources/legal-policy-analyses/107-the-narcotic-drugs-and-psychotropic-substances-control-act2015-and-the-legal-regulation-of-drug-use-in-uganda/file

2. Abbott, F. M., Abbott, R., Fortunak, J., Gehl Sampath, P., \& Walwyn, D. J. N. W. (2021). Opportunities, Constraints and Critical Supports for Achieving Sustainable Local Pharmaceutical Manufacturing in Africa: With a Focus on the Role of Finance, Final Report.

https://doi.org/https://dx.doi.org/10.2139/ssrn.3811733

3. AFRICA, C. (2021, May, 7, 2021). Uganda: New Cannabis Regulations Finally Head to Cabinet; Will End Exclusive Israeli Export Arrangement. Retrieved September 9 from https://www.cannabiz-africa.com/uganda-new-cannabis-regulations-finally-head-to-cabinet-will-end-exclusive-israeli-exportarrangement/ 
4. Cannabis for Private Purposes Bill (2020).

https://www.parliament.gov.za/storage/app/media/Bills/2020/B19_2020_Cannabis_for_Private_Purposes_Bill/B19_2020_Cannabis_for_Private_Purpose:

5. Aliekperova, N., Kosyachenko, K., \& Kaniura, O. J. J. o. C. R. (2020). Perspectives on formation of medical cannabis market in Ukraine based on holistic approach. 2(1), 1-19.

6. Ane, M.-G. (2020). Parliament of Ghana passes historic new drug law, paving the way for a West African approach. Retrieved 18 September from https://idpc.net/blog/2020/04/parliament-of-ghana-passes-historic-new-drug-law-paving-the-way-for-a-west-african-approach

7. Beadle, A. (2021, 02 July, 2021). Rwanda Legalizes Medical Cannabis. Retrieved 05 September from https://www.analyticalcannabis.com/news/rwandalegalizes-medical-cannabis-313177

8. BENDU, S. M. D. (2017, 02 February 2017). Cannabis legalisation in Sierra Leone, what hope? Retrieved November 27 from https://idpc.net/alerts/2017/02/cannabis-legalisation-in-sierra-leone-what-hope

9. Bizimungu, J. (2020). What production of medical cannabis means for the Rwandan economy? The New Times. Retrieved 18 October 2020 from https://www.newtimes.co.rw/news/what-production-medical-cannabis-means-rwandan-economy

10. Bloomer, J. (2019). Turning Cannabis Into Cash: Agrarian Change and Lesotho's Evolving Experience. EchoGéo(48). https://doi.org/10.4000/echogeo.17612

11. Carrier, N., \& Klantschnig, G. (2017). Quasilegality: khat, cannabis and Africa's drug laws. Third World Quarterly, 39(2), 350-365. https://doi.org/10.1080/01436597.2017.1368383

12. Chan, G. C., Leung, J., Quinn, C., Weier, M., \& Hall, W. J. A. (2018). Socio-economic differentials in cannabis use trends in Australia. 113(3), $454-461$.

13. Chisaka, J. W. (2019). The use of traditional herbal medicines among palliative care patients at Mulanje Mission Hospital, Malawi Faculty of Health Sciences].

14. Conway, M., Holt, T., Sabow, A., \& Sun, I. Y. (2020). Should sub-Saharan Africa make its own drugs. In: Retrieved.

15. Dirisu, O., Shickle, D., \& Elsey, H. (2018). Perspectives of young cannabis users within the Nigerian policy context: The paradox of criminalization as a deterrence strategy. Journal of Substance Use, 24(1), 73-78. https://doi.org/10.1080/14659891.2018.1510052

16. Eligh, J. (2019). The Evolution of Illicit Drug Markets and Drug Policy in Africa. https://enact-africa.s3.amazonaws.com/site/uploads/2019-06-30continental-report-3-3.pdf

17. Gebremariam, T. B., Mruts, K. B., \& Neway, T. K. (2018). Substance use and associated factors among Debre Berhan University students, Central Ethiopia. Substance Abuse Treatment, Prevention, and Policy, 13(1), 13. https://doi.org/10.1186/s13011-018-0150-9

18. Greenfield, T. K., Johnson, S. P., \& Giesbrecht, N. (2004). Public Opinion on Alcohol Policy: A Review of U.S. Research. 31(4), 759-790. https://doi.org/10.1177/009145090403100408

19. Jain, R., Chang, C. C., Koto, M. A., Geldenhuys, A. N., Nichol, R. J., \& Joubert, G. (2018). Cannabis use and knowledge among medical students at the University of the Free State, Bloemfontein, South Africa. J Child Adolesc Ment Health, 30(1), 19-26. https://doi.org/10.2989/17280583.2018.1438291

20. Jasmine Turna, I. B., Michael Van Ameringen, Jason W. Busse, and James Mackillop. (2020). Attitudes and Beliefs Toward Cannabis Before Recreational Legalization: A Cross-Sectional Study of Community Adults in Ontario. Cannabis and Cannabinoid Research. https://doi.org/https://doi.org/10.1089/can.2019.0088

21. Lintzeris, N., Mills, L., Suraev, A., Bravo, M., Arkell, T., Arnold, J. C., Benson, M. J., \& McGregor, I. S. (2020). Medical cannabis use in the Australian community following introduction of legal access: the 2018-2019 Online Cross-Sectional Cannabis as Medicine Survey (CAMS-18). Harm Reduction Journal, 17(1), 37. https://doi.org/10.1186/s12954-020-00377-0

22. Luty, J., \& Grewal, P. (2002). A survey of the British public's attitudes towards drug dependence. Journal of Substance Use, 7(2), 93-95. https://doi.org/10.1080/14659890210132126

23. Mametja, M., \& Ross, E. (2020). Decriminalized, Not Legalized: A Pilot Study of South African University Students' Views on the Use, Impact, Legalization and Decriminalization of Marijuana. 50(4), 490-506. https://doi.org/10.1177/0022042620931480

24. Manu, E., Douglas, M., Ntsaba, M. J., Tarkang, E. E. J. S. a. t., prevention, \& policy. (2021). Perspectives of illicit marijuana growers and traders on commercial legalisation of marijuana in South Africa: considerations for policy formulation. 16(1), 1-13.

25. Mehanović, E., Virk, H. K., Akanidomo, I., Pwajok, J., Prichard, G., van der Kreeft, P., \& Vigna-Taglianti, F. (2020). Correlates of cannabis and other illicit drugs use among secondary school adolescents in Nigeria. Drug and Alcohol Dependence, 206, 107457. https://doi.org/https://doi.org/10.1016/j.drugalcdep.2019.04.028

26. Meier, E., Tessier, K. M., Luo, X., Dick, L., Thomson, N. M., Hecht, S. S., Carmella, S. G., Murphy, S., \& Hatsukami, D. K. (2021). Cigarette Smokers Versus Cannabis Smokers Versus Co-users of Cigarettes and Cannabis: A Pilot Study Examining Exposure to Toxicants. Nicotine \& Tobacco Research. https://doi.org/10.1093/ntr/ntab125

27. Mendiburo-Seguel, A., Vargas, S., Oyanedel, J. C., Torres, F., Vergara, E., \& Hough, M. J. I. J. o. D. P. (2017). Attitudes towards drug policies in Latin America: results from a Latin-American Survey. 41, 8-13.

28. Mikos, R. A., \& Kam, C. D. (2019). Has the "M" word been framed? Marijuana, cannabis, and public opinion. PLOS ONE, 14(10), e0224289. https://doi.org/10.1371/journal.pone.0224289

29. Naidoo, P. (2021, 24 August 2021). South Africa's unemployment rate is now highest in the world. Retrieved 27 November from https://www.aljazeera.com/economy/2021/8/24/south-africas-unemployment-rate-is-now-the-worlds-highest

30. Nelson, E. U. (2018). Police crackdowns, structural violence and impact on the well-being of street cannabis users in a Nigerian city. Int J Drug Policy, 54, 114-122. https://doi.org/10.1016/j.drugpo.2018.01.012 
31. Onifade, A. J. A. J. o. M., \& Sciences, M. (2020). The use of herbal medicine for treatment of HIV infection. 49(2), $163-171$.

32. Owusu, N. O., Arthur, B., \& Aboagye, E. M. (2021). Industrial hemp as an agricultural crop in Ghana. Journal of Cannabis Research, 3(1), 9. https://doi.org/10.1186/s42238-021-00066-0

33. Partners, P. (2019). Africa Cannabis Report. https://kkog.global/wp-content/uploads/2019/10/The-African-Cannabis-Report\%E2\%84\%A2.pdf

34. Peltzer, K., \& Pengpid, S. (2018). Cannabis and Amphetamine Use and Associated Factors Among School-Going Adolescents in Nine African Countries. Journal of Child \& Adolescent Substance Abuse, 27(2), 112-118. https://doi.org/10.1080/1067828X.2017.1420512

35. Piontek, D., Kraus, L., Bjarnason, T., Demetrovics, Z., \& Ramstedt, M. J. J. O. A. H. (2013). Individual and country-level effects of cannabis-related perceptions on cannabis use. A multilevel study among adolescents in 32 European countries. 52(4), 473-479.

36. Putri, D. (2020). Cannabis rescheduling: What could it mean for Africa?

37. Resko, S., Ellis, J., Early, T. J., Szechy, K. A., Rodriguez, B., Agius, E. J. S. u., \& misuse. (2019). Understanding public attitudes toward cannabis legalization: qualitative findings from a statewide survey. 54(8), 1247-1259.

38. Robertson, K. J., Tustin, K. J. S. a. r., \& treatment. (2020). Control of recreational cannabis in a New Zealand university sample: perceptions of informal and formal controls. 14,1178221820953397.

39. Salehi, B., Kumar, N. V. A., Şener, B., Sharifi-Rad, M., Kılıç, M., Mahady, G. B., Vlaisavljevic, S., Iriti, M., Kobarfard, F., Setzer, W. N., Ayatollahi, S. A., Ata, A., \& Sharifi-Rad, J. (2018). Medicinal Plants Used in the Treatment of Human Immunodeficiency Virus. 19(5), 1459. https://www.mdpi.com/14220067/19/5/1459

40. Skliamis, K., Benschop, A., \& Korf, D. J. (2020). Cannabis users and stigma: A comparison of users from European countries with different cannabis policies. O(0), 1477370820983560. https://doi.org/10.1177/1477370820983560

41. Sowoya, L., Akamwaza, C., Matola, A. M., Klein, A. J. D., \& Today, A. (2020). Goodbye Nicky hello Goldie-exploring the opportunities for transitioning tobacco farmers into cannabis production in Malawi. https://www.emerald.com/insight/content/doi/10.1108/DAT-04-2020-0021/full/pdf?title=goodbyenicky-hello-goldie-exploring-the-opportunities-for-transitioning-tobacco-farmers-into-cannabis-production-in-malawi

42. Steigerwald, S., Cohen, B. E., Vali, M., Hasin, D., Cerda, M., \& Keyhani, S. J. J. o. a. m. (2020). Differences in opinions about marijuana use and prevalence of use by state legalization status. 14(4), 337.

43. Sznitman, S. R., \& Bretteville-Jensen, A. L. J. H. r. j. (2015). Public opinion and medical cannabis policies: examining the role of underlying beliefs and national medical cannabis policies. 12(1), 1-10.

44. Tamson, M., Vorobjov, S., Sokurova, D., \& Pärna, K. (2021). Cannabis use and associated factors among 15-16-year-old adolescents in Estonia 20032019: Results from cross-sectional ESPAD surveys. Nordic Studies on Alcohol and Drugs, 38(3), 293-304. https://doi.org/10.1177/14550725211003415

45. Tharoor, A. (2018). South Africa decriminalises personal cannabis use and cultivation. Talking Drugs. Retrieved 28 October 2020 from https://idpc.net/alerts/2018/10/south-africa-decriminalises-personal-cannabis-use-and-cultivation

46. Turna, J., Balodis, I., Van Ameringen, M., Busse, J. W., MacKillop, J. J. C., \& Research, C. (2020). Attitudes and Beliefs Toward Cannabis Before Recreational Legalization: A Cross-Sectional Study of Community Adults in Ontario.

47. United Nations Office on Drugs and Crime, E. C. O. W. A. S., the European Union (2019). WENDU Report Illicit Drugs and Supply (2014-2017). https://fr.africacheck.org/wp-content/uploads/2020/03/WENDU_REPORT_VF_V5_291019_FINAL_VERSION.pdf

48. Veldman, M. (2020a, 06/19/2020). Cannabis in Sierra Leone - Laws, Use, and History. Sensi Seeds. Retrieved 19 October 2020 from https://sensiseeds.com/en/blog/countries/cannabis-in-sierra-leone-laws-use-history/

49. Veldman, M. (2020b, 06/19/2020). Cannabis in South Africa - Laws, Use, and History. Sensi Seeds. Retrieved 19 October 2020 from https://sensiseeds.com/en/blog/countries/cannabis-in-south-africa-laws-use-history/?sqr=south\%20africa

50. Williams, J., van Ours, J. C., \& Grossman, M. (2016). Attitudes to legalizing cannabis use. 25(9), 1201-1216. https://doi.org/https://doi.org/10.1002/hec.3340

\section{Figures}


(a)

Perceived risks of Cannabis use

\begin{tabular}{|c|c|c|c|}
\hline Ghana & $29.9 \%$ & $39.2 \%$ & $27.4 \%$ \\
\hline Nigeria & $29.4 \%$ & $38.4 \%$ & $31.3 \%$ \\
\hline Sierra Leone & $33.8 \%$ & $41.3 \%$ & $22.9 \%$ \\
\hline South Africa & $35.2 \%$ & $35.8 \%$ & $27.3 \%$ \\
\hline Uganda & $29.2 \%$ & $37.6 \%$ & $31.8 \%$ \\
\hline Zimbabwe & $32.2 \%$ & $36.2 \%$ & $31.0 \%$ \\
\hline
\end{tabular}

Social \& legal problems Health risks OSubstance abuse No risk

(b) Perceived benefits of Cannabis use

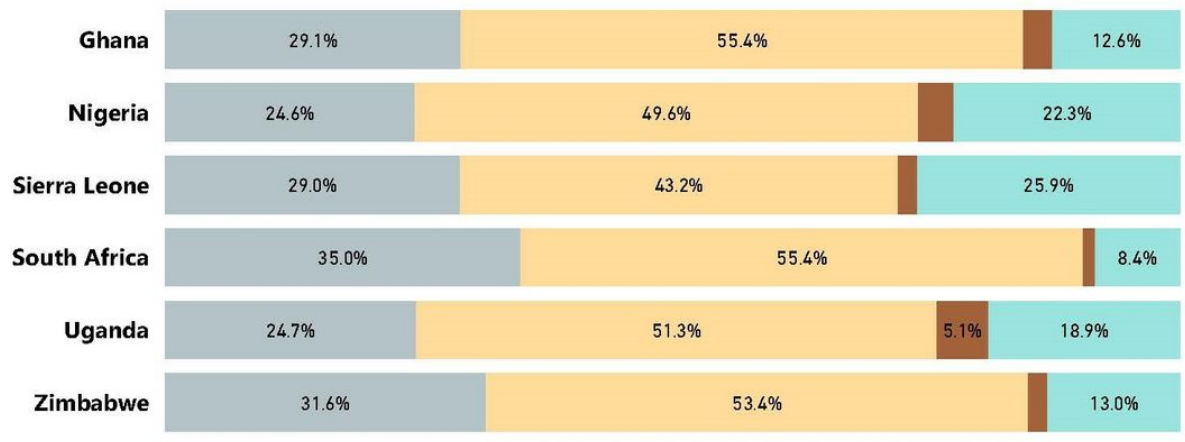

Social benefits Health benefits Other benefits No benefit

Figure 1

Figure 1- Perceived risks and benefits of Cannabis use 
(a) What do you think should be the legal status of cannabis?

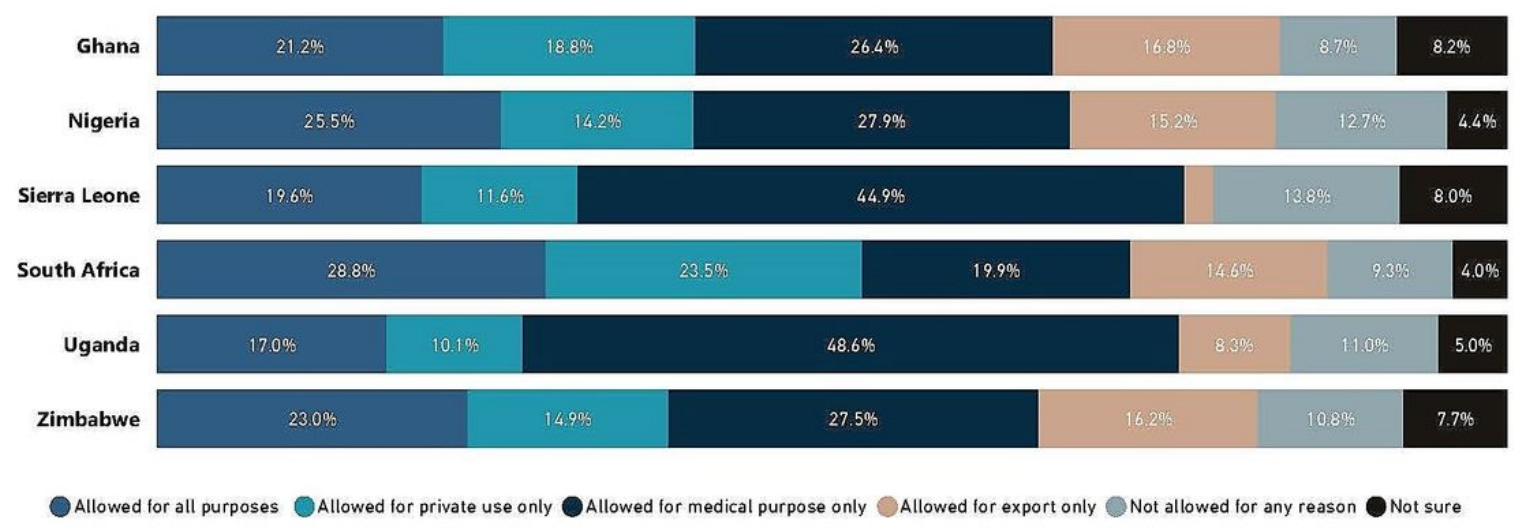

(b)

\section{People who use cannabis should be}

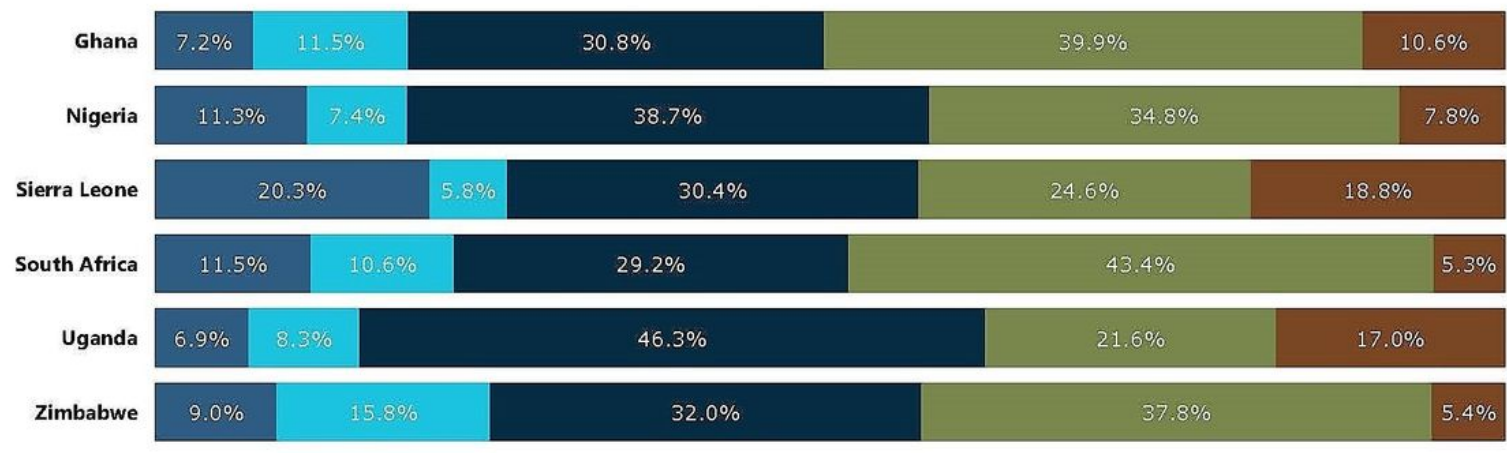

Arrested Fined Sent for treatment or counselling Left alone Other

Figure 2

Figure 2- Preference of policy approach and adressing users

\section{Supplementary Files}

This is a list of supplementary files associated with this preprint. Click to download.

- SupplementaryFile1Survey.pdf 